\title{
The Gardner syndrome: a cell culture study on Kindred 109
}

\author{
B. SHANNON DANES ${ }^{1}$ AND ELDON J. GARDNER
}

From the Laboratory for Cell Genetics, Department of Medicine, Cornell University Medica! College, New York New York 10021; and the Department of Biology, Utah State University, Logan, Utah, USA

SUMMARY In vitro studies on skin cultures established from 49 members from Kindred 109, in whorf the Gardner syndrome was first delineated, showed that increased in vitro tetraploidy occurred only if $\vec{p}$ those cultures derived from branches with the full expression of the Gardner gene (colorectal polypes with multiple extracolorectal benign tumours) and not in those derived from branches showing onl䓂 extracolorectal lesions. Increased in vitro tetraploidy appeared to identify only those family members at risk who had, or would ultimately be expected to show, full expression of the Gardner geneincluding colorectal polyps which become malignant.

Endoreduplication with increased tetraploidy has been observed (Danes, 1976b, 1978) in skin and colonic mucosa cultures containing epithelioid cells derived from patients with the autosomal dominant Gardner syndrome (association of multiple colorectal polyps which become malignant with cystic lesions of the skin, fibrous tissue tumours, and osteomatosis in a single family group) (Gardner, 1962, 1972; Pierce, 1972). As increased tetraploidy was observed only in cultures derived from these two tissues, which are known to show malignancies, and not in those derived from connective tissues, which show either normal or benign growths (Danes, 1976b), it has been proposed that increased tetraploidy in vitro does not identify cells with the Gardner genotype, but rather those that are known to undergo: malignant transformation in vivo (Danes, 1978).

Through detailed pedigree studies it has become recognised that within certain Gardner syndrome families some branches have variable extracolorectal lesions in association with colorectal growths, whereas in other branches the extracolorectal lesions may be present without the clinical expression of colorectal polyps or the history of colorectal cancer in consecutive generations. Kindred 109, in whom Gardner

'Supported by Public Health Service Grant CA-15973 from the National Cancer Institute through the National Large Bowel Cancer Project, and in part by the Danes Medical Research Fund, Cornell University Medical College, and the Zemurray Foundation.

2Supported by Grant VC-441 from the American Cancer Society, Utah State University Affiliated Exceptional Child Center, and Utah State University Biology Department.

Received for publication 21 November 1977 et al. (1952) and Gardner $(1962,1972)$ delineated this syndrome, is such a family group now extended through several generations (Fig.).

Determination of the occurrence of tetraploidy i skin cultures derived from clinically affected members who belong to branches of Kindred 109 who show fut expression (colorectal lesions and benign extr\% colorectal growths) and partial expression (only extrą colorectal benign lesions) should test the hypothesict that tetraploidy identifies in vitro a population of cells which are known to undergo malignant transo formation in vivo.

\section{Materials and methods}

Skin biopsies were obtained from a total of $4 \Phi$. members of Kindred 109 (Fig., Table): 6 with the fu愚 expression of the Gardner gene, 8 with partia expression (extracolorectal lesions), 6 who appeare clinically normal and were offspring of members showing full gene expression, 21 normals who were offspring of members showing partial or no expression of the Gardner gene, as well as 8 members byer marriage. Cell cultures were established from these split-thickness biopsies by standard culture methods (Danes and Bearn, 1969). Cultures were grown in Falcon plastic petri dishes in Eagle's minimum essential medium with $20 \%$ newborn calf serum and $5 \% \mathrm{CO}_{2}$ in air. The $\mathrm{pH}$ of the medium was kep\$ between 7.0 and 7.4 during the culture period.

After 2 culture weeks the initial explant culture was examined microscopically to verify that both sheets of 


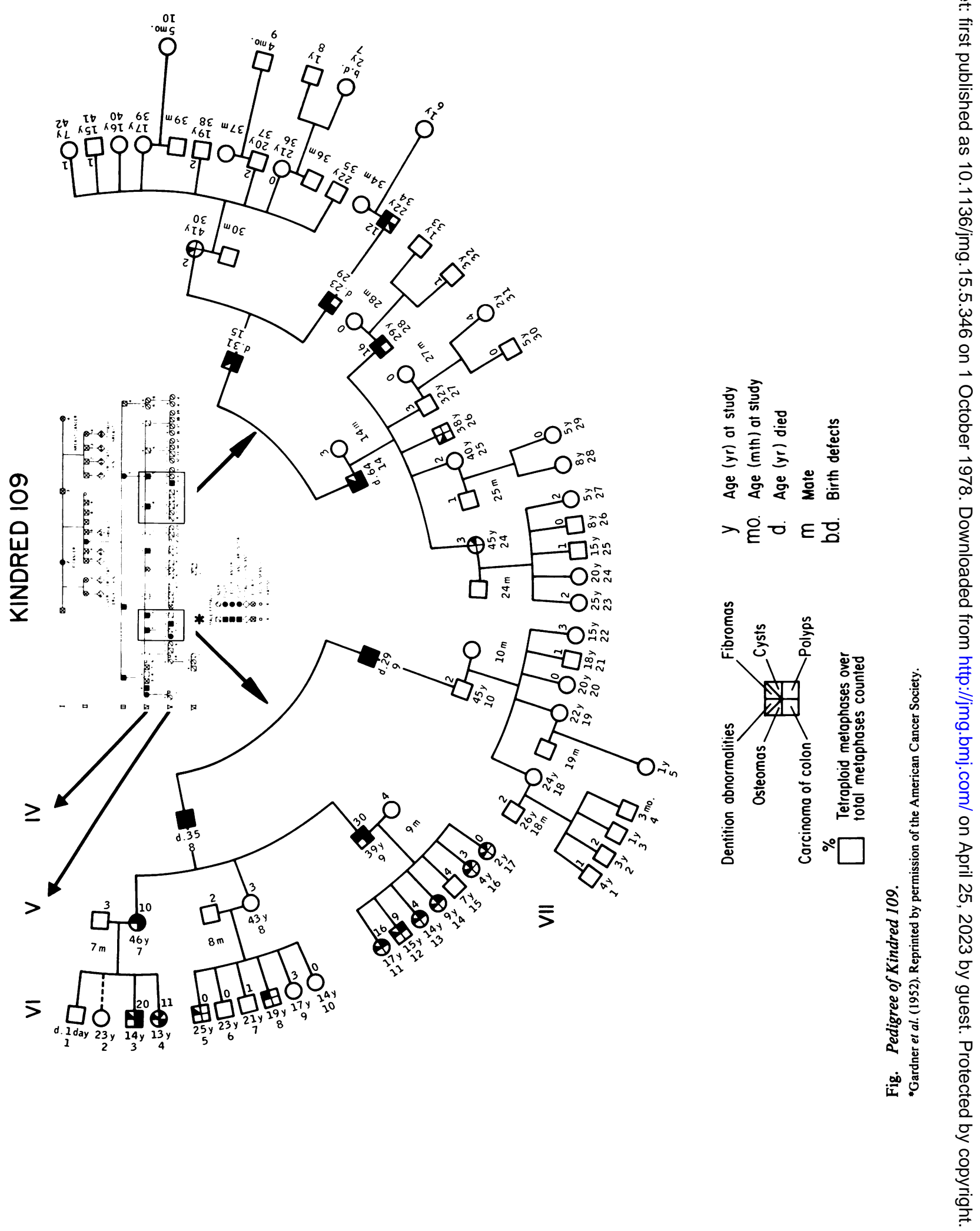


Table Percentage of dividing cells showing tetraploidy in skin cultures from 41 members of the Kindred 109 (Gardner et aDe 1952; Gardner, 1972) and 8 members by marriage

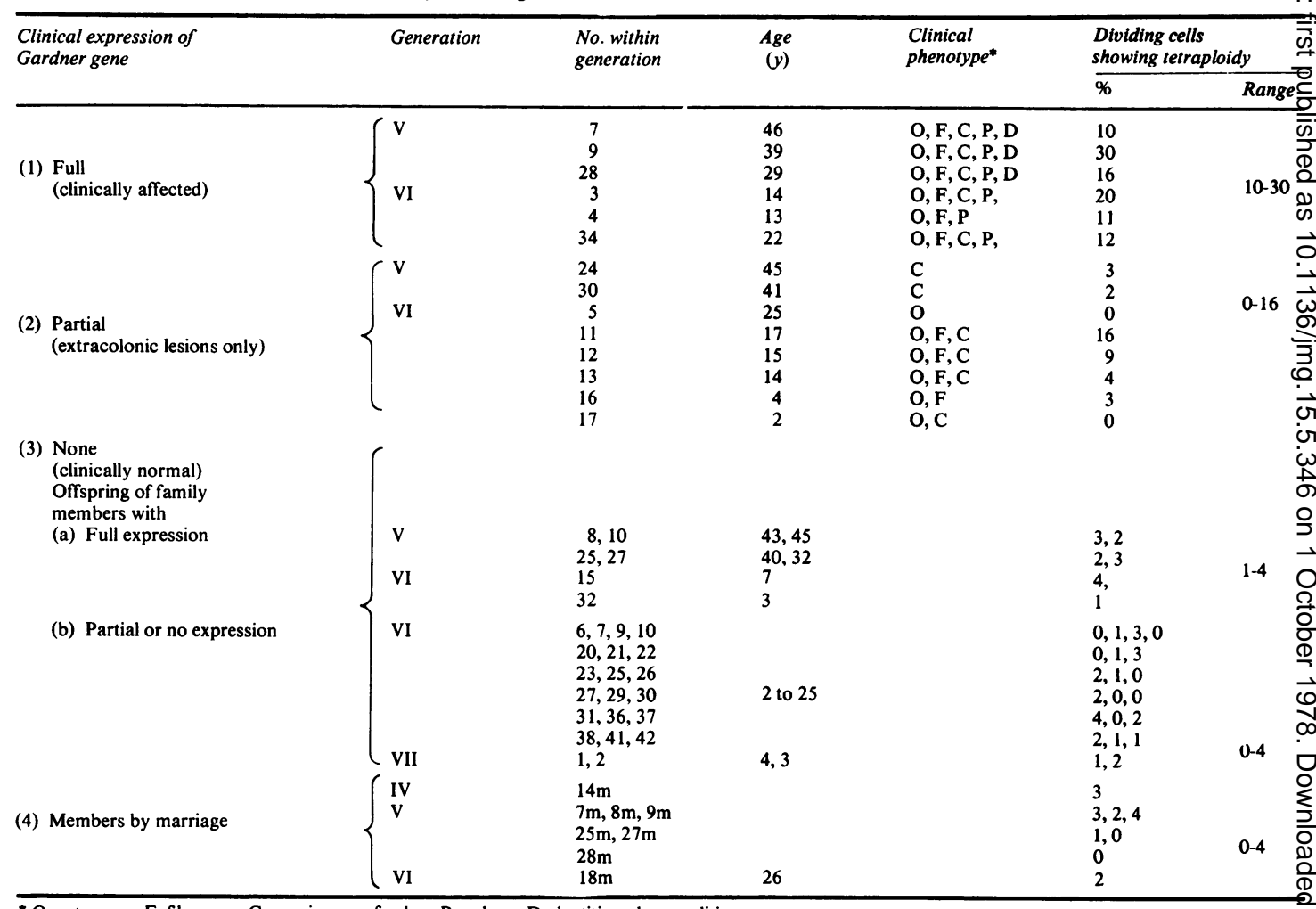

* O, osteomas; F, fibromas; C, carcinoma of colon; P, polyps; D, dentition abnormalities

epithelioid cells, presumably from the epidermis, and fibroblasts from the dermis were present in the migration zone surrounding the explant before trypsinisation to obtain a cell line. The cells were grown in culture 3 to 12 weeks ( 2 to 5 subcultures by trypsinisation) before the reported studies. Chromosome preparations were made and evaluated for tetraploidy as previously described in detail (Danes, 1976a). For each biopsy cultured, chromosome preparations were made on 2 different cultures from 2 sublines, and mitoses on 2 slides from each chromosome preparation were evaluated. As it was considered that a slide must contain 50 mitoses to reflect the mitotic activity of a cell strain, slides with less than 50 mitoses were not included in this study.

The occurrence $(\%)$ of tetraploidy in a culture was expressed as the number of metaphases showing tetraploidy divided by the total number of metaphases counted.

\section{Observations}

Based on family history and clinical examinations in generations V to VII (Fig.), the clinical phenotype of the Gardner syndrome in Kindred 109 was multiple colorectal polyps which become malignant and extras colorectal abnormal connective tissue growth (osteomas, dentition abnormalities, fibromas, and cysts). The 3 affected members studied in generation $\bar{B}$ (nos. 7, 9, 28; ages 46, 39, 29) had the same clinicat expression (osteomas, fibromas, cysts, polyps, an $\Phi$. dentition abnormalities ). In generation VI, the extra? colorectal lesions in VI.4 (first observed at age 4) were osteomas and fibromas, whereas in VI.3 and VI.34 (ages 14 and 22) cysts were also present. The skin cultures established from all 6 members with the fut expression of the Gardner syndrome showed increase tetraploidy (Table). The percentage of cells in metaer phase showing tetraploidy ranged from 10 to 30 , was constant for each line through repeated subcultures and did not appear to be influenced by the age of the donor. The number of chromosomes per cell in suck cells was tetraploid (92) or hypotetraploid.

One of these patients (V.9) had 7 offspring: (VI.11, 12, 13, 16, 17) of the 6 studied had multiple extracolorectal lesions. The cultures from 2 (VI.11.12 ; ages 17,15$)$ showed increased tetraploidy $(16 \% \overrightarrow{\mathrm{Q}}$ $9 \%)$, whereas those from the other 3 (VI.13, 16, 17 
ages $14,4,2)$ did not $(4 \%, 3 \%, 0 \%)$. The other offspring (VI.15, age 7) studied had a normal clinical phenotype and his cultures showed no increase in tetraploidy (4\%).

In generation $\mathrm{V}$ the cultures from 2 members (V.24, 30; ages 45,41 ) with partial expression (cysts) showed no increase in tetraploidy $(3 \%, 2 \%)$. In generation VI the offspring (VI.23-27) of V24 and (VI.35-42) of V.30, (ages 5 to 25) had normal phenotypes; the cultures of the 9 studied showed no increased tetraploidy ( 0 to $2 \%$ ).

One member (V.8) did not show any clinical evidence of having inherited the Gardner gene and her cell cultures showed no increased tetraploidy (3\%). Of her 6 offspring, 2 had an extracolonic lesion (VI.5, age 25 , and VI.8, age 19, each had a lesion that appeared as an osteoma and VI.8 also had dentition abnormalities). A biopsy was obtained only from VI.5, which yielded cultures showing no increased tetraploidy. The cultures established from her 4 other offspring (VI.6, $7,9,10$, ages 14 to 23 ) who did not have any clinical evidence of the Gardner gene had no increase in tetraploidy (0 to $3 \%$ ).

Three members $(V .10,25,27)$ had no clinical signs of the Gardner syndrome at ages 45,40 , and 32 years, respectively, and their cultures showed $2 \%, 2 \%$, and $3 \%$ tetraploidy. Six (VI.20, 21, 22, 29, 30, 31; ages 2 to 20 years) of their 9 normal-appearing offspring were studied and none of their cell cultures showed increased tetraploidy ( 0 to $4 \%$ ). The cultures from the 2 grandchildren of V.10 (VII.1, 2; ages 4 and 3 years) also showed no increase in tetraploidy $(1 \%, 2 \%)$, neither did those derived from 8 members by marriage in 3 generations ( 0 to $4 \%$ ).

\section{Discussion}

In an autosomal dominant disorder such as the Gardner syndrome in which there are age dependent manifestations (colorectal polyps and neoplasms) and extracolorectal lesions (Gardner, 1972; Pierce, 1972), a consistent cell abnormality identifying the Gardner gene would help in recognition of family members who have inherited the gene before clinical signs appear, so that appropriate medical surveillance and genetic counselling could be initiated.

In endoreduplication, chromosome replication occurs without an intervening mitosis resulting in a tetraploid nucleus. Endoreduplication with resultant tetraploidy has been reported to occur in cultured cells from normal individuals but at a very low incidence (Puck et al., 1958; Obe, 1965; Turner and Wald, 1965; Todaro and Martin, 1967; Danes, 1976a, b, 1978). In the present study (Fig., Table) the occurrence of tetraploidy in cultures established from 27 kindred members showing no clinical stigmata of the
Gardner syndrome and 8 members by marriage was low (0 to $4 \%)$.

When endoreduplication with increased tetraploidy was first observed in skin cultures from patients with the Gardner syndrome, and not from those derived from patients with other heritable syndromes having one of the lesions associated with the Gardner syndrome, it was proposed that increased tetraploidy might detect the Gardner gene in vitro (Danes, 1975, 1976a). Subsequent research determined that increased tetraploidy did not identify all cultured cells with the Gardner gene, as fibroblast cultures irrespective of their tissue source and white blood cells showed none, neither did it identify cultured cells from abnormal benign tumours, as cultures established from fibromas and cysts did not show any such mitotic abnormality (Danes, 1976b). Increased tetraploidy as an expression of the gene for the Gardner syndrome occurred only in cultures from epithelial-containing tissues which are known to undergo malignant transformation in vivo, epidermis and colonic mucosa.

Colonic polyps without evidence of neoplastic changes from patients with the Gardner syndrome have been reported to show chromosome abnormalities including tetraploidy (Mark et al., 1973). It has thus been assumed that such aberrant mitoses occurred in the epithelioid cell with the Gardner syndrome genotype, both in vivo and in vitro.

In a kindred with the Gardner syndrome such as Kindred 109, such a cell marker would be of value for 3 kinds of family groups (those with full, partial, or no expression of the Gardner gene) (Fig., Table).

The first family group to have in vitro studies performed was that with full expression, 6 members in which the diagnosis had been made on the basis of the full clinical phenotype. The finding that the skin cultures established from their biopsies all showed increased tetraploidy only confirmed the clinically established diagnoses. These data supported the observation that increase in vitro tetraploidy occurs in cultures established from the epidermis of patients showing the full expression of the Gardner gene (Danes, 1976b).

The second family group in which in vitro studies were considered to be of interest was that showing only partial expression (extracolorectal lesions), as it could not be established on clinical criteria whether colorectal lesions would occur later in life. As it has been reported that the time interval between the first soft tissue tumours and the diagnosis of the Gardner syndrome based on bowel symptoms occurring secondary to colorectal polyps was 17 years (Duncan et al., 1968), a test which detected if the Gardner gene would eventually be expressed in the colon of such a patient with only extracolorectal lesions would be of value in clinical management. 
In Kindred 109 (Gardner, 1972) there was a family history of adult members showing only extracolorectal lesions without colorectal lesions. Colonic polyps in this family were usually found on colonoscopy in the early teens with the earliest appearance of colon polyps (in V.9) at age 13. He had been examined at regular 6-monthly intervals from age 11 until the appearance of the first polyp at $6 \mathrm{~cm}$ when he was aged 13. At age 17, V.9 had multiple colorectal polyposis. A colectomy was performed at that time with the stump of the rectum left intact. Polyps have been removed from the rectum of V.9 at regular intervals over the past 22 years.

Eight family members were considered to show only partial expression. Two (V.24, 30) were old enough (45 and 41 years) to have shown colonic lesions and their cultures showed no increased tetraploidy. VI..5 (age 25) had an apparent osteoma on his mandible and no increased in vitro tetraploidy $(0 \%)$. His mother (V.8) did not show any clinical or in vitro expression ( $3 \%$ tetraploidy) of the Gardner gene. The 6 other members in generation VI who had extracolorectal manifestations without colorectal lesions were the offspring of V.9, a 39-year-old man with full expression of the Gardner syndrome and $30 \%$ in vitro tetraploidy. The cultures from his 2 oldest offspring (VI.11, 12; ages 17, 15 years) showed increased tetraploidy $(16 \%, 9 \%)$ and thus it was considered that they would eventually show full expression. If the appearance of polyps is similar to that of their father (V.9) and other members of Kindred 109, they will now have colorectal polyps. VI.11 and 12 will be examined in the very near future.

The absence of increased tetraploidy in skin cultures from the 3 youngest offspring investigated (VI.13, 16, 17 ; ages $14,4,2$ years) with extracolorectal lesions might have been due to one of three possibilities. (1) The occurrence of tetraploidy in cultured skin cells might be age-dependent as the occurrence of in vivo polyps is known to be (Gardner, 1972; Pierce, 1972). However, increased in vitro tetraploidy has been reported in skin cultures derived from clinically asymptomatic members at risk in other families with the Gardner syndrome as early as 5 years of age (Danes and Krush, 1977). Cultures established from repeat biopsies from the 3 younger offspring over the next years should determine if chronological age or first appearance of colorectal polyps influenced the occurrence of in vitro tetraploidy. (2) The possibility that epithelioid cells from the epidermis had not been included in the cell population studied from the 3 younger offspring in question must be considered. Until pure epidermal cultures can be established, variation in the proportion of epidermal cells to fibroblast cells, which have been shown not to show increased tetraploidy (Danes, 1976b), included in the cell sample studied could produce erroneously low in cidence of tetraploidy. Establishment of subculturesD from repeat skin biopsies should answer this question (3) Increased tetraploidy may be expressed in skins? cultures derived only from those individuals with theo Gardner gene who will ultimately develop colorectaof polyps, and if not treated, cancer.

As colonic mucosa from patients with the Gardner syndrome shows increased in vitro tetraploidy (Danes, 1976b), cultures of colonic mucosa should be studied? from all of the offspring with extracolorectal lesions. I $\vec{P}$ cannot be assumed that the 3 youngest offspring with $\overrightarrow{-}$ extracolorectal lesions and no increased tetraploidy in $\omega$ their skin cultures will not develop colonic polyps untis all three of these possibilities have been evaluated.

The absence of increased tetraploidy in familyer members known to have the Gardner gene with partiai clinical expression supported the hypothesis that increased tetraploidy does not identify the Gardners genotype but rather identifies in vitro a population of cells which are known to undergo malignant trans $-\overrightarrow{0}$ formation in vivo. Such observations suggest thats there is a population of tetraploid cells having chromo음 somal instability, at least in culture, constantly presen $\mathbb{C}^{\circ}$ which may be relevant to the multi-step process of carcinogenesis (Knudson et al., 1973; Knudson 1977).

The third family group (V.8 and her children VI.5, $6,7,8,9,10)$ to be studied by cell culture methodology was at risk to inherit the Gardner gene but showed no clinical signs, irrespective of age. Noneo showed increased in vitro tetraploidy. Further study is required. The most likely interpretation of the presen $\overrightarrow{\overrightarrow{6}}$ data is that V.8 (with a $50 \%$ risk) missed the gene for the disorder and that she and her 6 children do no carry the Gardner gene. Two of her children (VI.5, 8) however, developed discrete solitary lesions, similar tos those of the Gardner syndrome, in their late teens an $\bar{P}$ early twenties. This family group has been studied extensively along with those of V.7 and V.9 over $\vec{b}$ period of 30 years. During this period multiple osteomas, fibromas, epidermoid cysts, and polyps have been detected in VI.3 and 4 and multiple extra은 colonic lesions in VI.11, 12, 13, 14, 16, 17. These are confirmed histologically. No expressions even suggest ing the Gardner syndrome were detected in V.8 and her family until recently. The most likely explanation for single, discrete, solitary lesions that appear to bes osteomas in VI.5 and the dental abnormalities in VI.\& is that they are related to trauma incurred in employ ment in the heavy car industry in which VI.5 and $\&$ are engaged. A similar explanation involving environ mental trauma may account for the isolated single cysts observed in V.24 and V.30.

In familial polyposis coli (Pierce, 1972), anothep autosomal dominant cancer syndrome, in which the? 
genotype is expressed solely as colorectal polyps that become malignant, with the skin showing no abnormalities, increased in vitro tetraploidy has been observed only in colonic mucosa and not in epidermis. Determination of the occurrence of tetraploidy in cultures established from colonic mucosal biopsies from members at risk in all 3 groups (those with full, partial, or no expression of the Gardner gene) in Kindred 109 would give valuable information concerning the gene expression in the colon, as the skin biopsies did for the epidermis (Fig., Table).

From the cell culture research on the Gardner syndrome (Danes, 1976a, b, 1978; Danes and Krush, 1977), including this study on Kindred 109, increased in vitro tetraploidy appeared to identify only those family members at risk who did or would ultimately show full expression of the Gardner gene.

This research was part of a collaborative study on heritable cancers as part of the National Large Bowel Cancer Project of the National Cancer Institute. Without the interest and enthusiasm of the Kindred 109 this research would not have been possible. The authors wish to acknowledge the reference work of M.F.D.

\section{References}

Danes, B. S. (1975). The Gardner syndrome. A study in cell culture. Cancer, 36, 2327-2333.

Danes, B. S. (1976a). The Gardner syndrome: increased tetraploidy in cultured skin fibroblast. Journal of Medical Genetics, 13, 5256.

Danes, B. S. (1976b). Increased tetraploidy: cell-specific for the Gardner gene in the cultured cell. Cancer, 38, 1983-1988.

Danes, B. S. (1978). Increased in vitro tetraploidy: tissue specific within the heritable colorectal cancer syndromes with polyposis coli. Cancer, 41. (In the press.)

Danes, B. S., and Bearn, A. G. (1969). Cystic fibrosis of the pancreas. A study in cell culture. Journal of Experimental Medicine, 129, 775-793.
Danes, B. S., and Krush, A. J. (1977). The Gardner syndrome: a family study in cell culture. Journal of the National Cancer Institute, 58, 771-775.

Duncan, B. R., Dohner, V. A., and Priest, J. H. (1968). The Gardner syndrome: need for early diagnosis. Journal of Pediatrics, 72, 497-505.

Gardner, E. J. (1962). Follow-up study of a family group exhibiting dominant inheritance for a syndrome including intestinal polyps, osteomas, fibromas and epidermal cysts. American Journal of Human Genetics, 14, 376-390.

Gardner, E. J. (1972). Discovery of the Gardner syndrome. Birth Defects: Original Article Series, 13, 48-51.

Gardner, E. J., Woolf, C. M., and Shaffer, J. O. (1952). The inheritance of cancer involving different areas of the human gastrointestinal tract. Proceedings of the Second National Cancer Conference, 1, 846-856.

Knudson, A. G., Jr. (1977). Mutation and cancer in man. Cancer, 39, $1882-1886$.

Knudson, A. G., Jr., Strong, L. C., and Anderson, D. E. (1973). Heredity and cancer in man. In Progress In Medical Genetics, Vol. 9, pp. 113-158, Ed. by A. G. Steinberg and A. G. Bearn. Grune and Stratton, London and New York.

Mark, J., Mitelman, F., Dencker, H., and Norryd, C. (1973). The specificity of chromosomal abnormalities in human colonic polyps. Acta Pathologica et Microbiologica Scandinavica, 81, 85-90.

Obe, G. (1965). Chromosome endoreduplication in normal controls. Lancet, 1, 53.

Pierce, E. R. (1972). Pleiotropism and heterogeneity in hereditary intestinal polyposis. Birth Defects: Original Article Series, 13, 52-62.

Puck, T. T., Steven, J. C., and Robinson, A. (1958). Genetics of somatic mammalian cells. II. Long-term cultivation of euploid cells from human and animal subjects. Journal of Experimental Medicine, 108, 945-956.

Todaro, G. J., and Martin, G. M. (1967). Increased susceptibility of Down's syndrome fibroblasts to transformation by $\mathrm{SV}_{40}$. Proceedings of the Society for Experimental Biology and Medicine, 124, 1232-1236.

Turner, J. H., and Wald, N. (1965). Endoreduplication and disease. Lancet, 1, 915.

Requests for reprints to Dr B. Shannon Danes, Department of Medicine, Laboratory for Cell Genetics, Cornell University Medical College, 1300 York Avenue, New York, NY 10021, USA. 